\title{
Breast restoration decision making followed 2 predominant patterns and did not involve active information seeking behaviour
}

Reaby LL. Breast restoration decision making: enhancing the process. Cancer Nurs 1998 Jun;21:196-204.

\section{Question}

What are women's decision making patterns about breast restoration after breast cancer treated by mastectomy?

\section{Design \\ \{Grounded theory\}*.}

\section{Setting}

The metropolitan area of Sydney, New South Wales, Australia.

\section{Patients}

95 women ( $80 \%$ white) who had breast cancer, modified radical mastectomy, and no clinical indications of recurrence were recruited by 3 general surgeons. 64 women (mean age $63 \mathrm{y}$ ) wore external breast prostheses and 31 women (mean age 49.5 y) had breast reconstructions.

\section{Methods}

A semistructured interview was conducted at the participant's home 2-7 years after the diagnosis of breast cancer. Interviews usually lasted about 50 minutes. Participants were asked about information seeking behaviour, and knowledge of and deliberation on breast restoration alternatives. Interviews were audiotaped, transcribed verbatim, and analysed by both the researcher and a clinical psychologist using the NUD•IST software programme. The breast restoration decision making process was categorised into 5 patterns based on Janis and Mann's decision making conflict model and Simon's notion of "bounded rationality": enlightened, which represented extensive information seeking behaviour and an active deliberation of the positive and negative aspects of the alternatives; contented, which described passive acceptance of information and a preference towards a certain alternative; sideliner, which identified an uncritical acceptance of the alternative that was easiest to implement; shifter, which characterised a woman with a high level of anxiety who shifted the decision to someone else; and panic stricken, which portrayed a woman in extreme stress who was unable to make a rational decision.

\section{Main findings}

Women who wore breast prostheses predominantly used the sideliner pattern of decision making and expressed regret after their decision. Fewer women with prostheses compared with those with reconstructions used either the contented pattern and had no regret after their decision, or the shifter pattern and expressed regret after their decision. Among women who had breast reconstructions, most used the contented pattern and a minority used the shifter pattern; and no regret after their decision was expressed. The enlightened pattern was not used by any of the women; and the sideliner pattern was not used by those who had breast reconstructions. The panic stricken pattern was used by 1 woman who had breast reconstruction.

\section{Conclusions}

In women who have had breast cancer treated by mastectomy, the breast restoration decision did not include active information seeking behaviour or deliberation of the alternatives. Most women who wore breast prostheses made the decision because it was the easiest alternative and most women who had breast reconstructions made the decision based on personal preference.

Source of funding: The Commonwealth Department of Human Services and Health; University of Canberra.

For correspondence: $\operatorname{Dr} L$ L Reaby, School of Nursing, University of Canberra, PO Box 1, Belconnen, ACT 2616, Australia. Fax +616062015128

*Information supplied by author

\section{Commentary}

Decisions made at critical times, such as during disease diagnosis and relapse, can have a profound effect on survival and quality of life. This study by Reaby is timely because many women are now being offered the choice of breast reconstruction at the time of mastectomy. Consistent with other research, women who had breast reconstruction were satisfied with their decision. ${ }^{1}$

The finding that some women who wore external breast prostheses were not satisfied with their decision prompted Reaby to design a model for competent breast restoration decision making. This model is intended to guide the information seeking process during a critical time for women in all age groups.

Although this is a well designed study, there is a large leap from the data to a highly structured model. This model, which has not been evaluated, is suggested for use before mastectomy. If used, it may cause anxiety for women, especially during the highly stressful time of diagnosis.

Reaby highlights that although breast reconstruction can offer solace to women, it is not a choice that is extended to everyone. Nurses need to recognise that whatever decision model is used, not all women will be satisfied with their outcomes even after they have been provided with knowledgeable answers and have completed a "competent" decision making process.

With modification, the model could have clinical applications for use during patient counselling and as a guide to support the nurses' advocacy role of ensuring their patients receive the optimal treatment.

Mary Brown, RN, RNT, MA Senior Lecturer in Nursing Studies Christ Church College University

Angela Wilson, RN, BSc (Hons) Breast Care Nurse Specialist Kent and Canterbury NHS Trust Canterbury, Kent, UK

1 Park AJ, Chetty U, Watson AC. Patient satisfaction following insertion of silicone breast implants. Br JPlast Surg 1996;49:515-8.

2 Neufeld KR, Degner LF, Dick JA. A nursing intervention strategy to foster patient involvement in treatment decisions. Oncol Nurs Forum 1993;20:631-5. 